\title{
Probabilistic Performance Analysis of Existing Buildings under Earthquake Loading
}

\section{Xavier Romão, Raimundo Delgado \& Aníbal Costa}

To cite this article: Xavier Romão, Raimundo Delgado \& Aníbal Costa (2014) Probabilistic Performance Analysis of Existing Buildings under Earthquake Loading, Journal of Earthquake Engineering, 18:8, 1241-1265, DOI: 10.1080/13632469.2014.937015

To link to this article: http://dx.doi.org/10.1080/13632469.2014.937015 Jun 2014 Published online: 25 Jun 2014.

Submit your article to this journal $₫$

Џلll Article views: 216

Q View related articles $\widetilde{ }$

View Crossmark data $[7$ 


\title{
Probabilistic Performance Analysis of Existing Buildings under Earthquake Loading
}

\author{
XAVIER ROMÃO ${ }^{1}$, RAIMUNDO DELGADO $^{1}$, \\ and ANÍBAL COSTA ${ }^{2}$ \\ ${ }^{1}$ Civil Engineering Department, Faculdade de Engenharia, Universidade do Porto, \\ Porto, Portugal \\ ${ }^{2}$ Civil Engineering Department, Universidade de Aveiro, Aveiro, Portugal
}

\begin{abstract}
A probabilistic methodology is proposed for the seismic performance analysis of existing buildings using global metrics to determine if the behavior conforms to a given limit state. The referred performance metrics are the mean annual frequency of the limit state, the corresponding expected loss associated to the repair of the building, and the corresponding number and type of mechanisms that occur. The consideration of these assessment parameters to control building performance widens the scope of the limit state definitions proposed in current codes. Therefore, current limit state descriptions were updated to establish adequate risk- and cost-related limit state definitions using the Eurocode 8 Part 3 proposals as a basis for discussion. The description of the proposed procedure is detailed and addresses its applicability for different limit states and its ability to include the uncertainty in the limit state capacities. An application involving the performance analysis of a reinforced concrete structure for several limit states is also presented and discussed.
\end{abstract}

Keywords Seismic Performance Assessment; Risk Analysis; Loss Assessment; Existing Buildings; Reinforced Concrete

\section{Introduction}

Probabilistic seismic safety assessment methods are intrinsic to the conceptual framework established by current Performance-Based Earthquake Engineering (PBEE) methodologies. This framework involves key aspects such as the use of adequate methods of analysis to determine building behavior and the definition of quantifiable targets to measure performance. In this context, the ATC-63 probabilistic methodology [Kircher and Heintz, 2008; Deierlein et al., 2008; ATC, 2009] was recently proposed. This methodology involves incremental dynamic analysis (IDA) [Vamvatsikos and Cornell, 2002] and probabilistic procedures to evaluate seismic fragility margins of the building system against collapse and to calibrate appropriate values of design-related parameters such as the behavior factor and other parameters affecting the response of the building. Although the main objective of this approach is to evaluate the seismic performance of new structures against collapse, thus determining the effectiveness of design rules established by current codes and standards, this methodology can also be adapted to assess the safety of existing structures [ATC, 2009].

Received 30 October 2013; accepted 17 June 2014.

Address correspondence to Xavier Romão, Civil Engineering Department, Faculdade de Engenharia, Universidade do Porto, Rua Dr. Roberto Frias, 4200-465 Porto, Portugal. E-mail: xnr@fe.up.pt

Color versions of one or more of the figures in the article can be found online at www.tandfonline.com/ueqe. 
The widespread interest in methodologies addressing the assessment and retrofit of existing constructions has led to the development of several normative documents and guidelines to assess their seismic performance (e.g., see ATC, 1996; ASCE, 2003; CEN, 2005; NZSEE, 2006; ASCE, 2007; NTC, 2008). The assessment methods proposed in these documents consist, essentially, of deterministic approaches involving the evaluation of the seismic response for different limit states which are quantitatively established by limit values of local (section level) demand parameters. These methods are expected to be reliable, since they may include modeling, analysis, and verification procedures more detailed than those commonly considered in the design of new structures. Nonetheless, a probabilistic validation of such safety assessment approaches appears to be essential to observe their adequacy. Given these considerations, the probabilistic approach presented herein is seen to be suitable for the evaluation of the seismic safety of existing structures according to the concepts found in recent codes and standards.

The proposed method enables practitioners to assess the probabilistic seismic safety of existing buildings and uses global performance metrics to determine if the building behavior conforms to a given limit state. The considered performance metrics are the mean annual frequency (and approximately the annual probability $\left.{ }^{\ddagger}\right) \lambda$ of exceeding the limit state, the corresponding expected loss $L_{s c}$ associated to the repair of the building, and the corresponding number and type of mechanisms that are developed and that establish possible scenarios for the occurrence of that limit state. The term mechanism is considered herein as referring to the occurrence of a limit state capacity in one or in a combination of several structural members [fib, 2003a; Pinto et al., 2004; Jalayer et al., 2007; LessLoss, 2007].

The consideration of $\lambda, L_{s c}$ and the occurrence of several mechanisms as global performance parameters for a given limit state requires an update of existing limit state descriptions. After analyzing the limit state descriptions of the seismic safety assessment procedures proposed in Part 3 of Eurocode 8 (EC8-3) [CEN, 2005], alternative proposals are discussed to establish risk- and cost-related limit state definitions. Although EC8-3 is used as a reference, the issues raised by this analysis of the limit states extend beyond this code since similar limit states or performance levels are also considered by other international standards.

The present article details the description of the proposed procedure and addresses its applicability for different limit states. The performance assessment of a reinforced concrete (RC) structure for several limit states is then presented and discussed.

\section{Probabilistic Performance Analysis Methodology}

\subsection{General Overview of the Methodology}

To analyze building performance for a given limit state, the proposed methodology uses the mean annual frequency $\lambda$ of the limit state, the corresponding loss $L_{s c}$ associated to the repair of the building, and the corresponding number of structural sections $n_{L S}$ where the limit state mechanism occurs. By setting a value for $n_{L S}$, a possible scenario for the occurrence of a given limit state is established, for which $\lambda$ and $L_{s c}$ are calculated. The values of $\lambda$ and $L_{s c}$ obtained for each of the $m_{s c}$ considered scenarios are then combined to obtain the expected loss $E L$ over a given reference period of time defined by:

${ }^{\ddagger}$ For events with a very small probability of occurrence, the numerical values of the mean annual frequency of occurrence and of the probability of occurrence in one year are very close. 


$$
E L=\sum_{i \in \Omega} \lambda_{i} \cdot L_{s c, i}, \Omega=\left\{i: L_{s c, i+1}>L_{s c, i}, i=1, \ldots, m_{s c}\right\}
$$

The constraint defined for the set $\Omega$ accounts for the possibility of having $m_{s c}$ scenarios which are not mutually exclusive. Such a situation can be found in structures where the $i t h$ scenario involving the occurrence of a given limit state in $n_{L S, i}$ sections and the $i t h+1$ scenario involving $n_{L S, i+1}$ sections lead to the same value of $L_{s c}$.

As in the Consequence-Based Engineering framework [Wen and Ellingwood, 2005], the value of the global performance metric $E L$ must be compared with an admissible limit $E L_{a d m}$ which defines the acceptable consequences of reaching a given limit state. In the proposed methodology, $E L_{a d m}$ is defined by:

$$
E L_{a d m}=\lambda_{a d m} \cdot L_{s c ~ a d m}
$$

where $\lambda_{a d m}$ and $L_{s c ~ a d m}$ are global acceptance thresholds defined for $\lambda$ and $L_{s c}$. The value of $\lambda_{\text {adm }}$ can be based on proposals recommended by existing standards and other technical documents [ISO 2394, 1998; JCSS, 2001; DOE, 2002; Diamantidis and Bazzurro, 2007; ISO 13822, 2010; Vrouwenvelder and Scholten, 2010]. With respect to $L_{s c}$ adm, although its value should also be bounded by code-based proposals, a case by case refinement might be required based on the stakeholders needs and expectations.

The value of $\lambda$ is estimated by

$$
\lambda=\int_{0}^{\infty} p_{f}(x) \cdot\left|\frac{d H(x)}{d x}\right| d x,
$$

where $H($.$) is the earthquake hazard curve defined in terms of a selected earthquake inten-$ sity measure (IM) and $p_{f}$ (.) is the fragility curve representing the probability of exceeding a given state of performance conditional to a certain value of the IM. The considered formulation of the fragility curve is similar to the IM-based approach referred in Ibarra et al [2002]. With respect to the expected value of the loss $L_{s c}$, its quantification is carried out using the story-based approach proposed by Ramirez and Miranda [2009], a procedure that has shown promising results (e.g., see Welch et al., 2014).

Although the potential total loss due to damage from earthquakes $L_{t o t}$ can be formulated in order to include different types of direct costs (costs due to structural damage, non structural damage, or loss of contents) and indirect costs (costs due to business interruption or injuries and fatalities), formulating all of them as a function of a unique engineering demand parameter (EDP) is not straightforward. For example, damage and costs can be more easily correlated to inter-story drift or to floor acceleration levels depending on the type of non structural element [Foltz, 2004]. Furthermore, since most of the indirect costs can be considered to be unique for each building, this requires an extensive and updated inventory which may not be available for the seismic safety analysis. In light of these considerations, a simplified loss model that only addresses losses due to structural and non structural damage, represented by $L_{s c}$, is considered herein.

When analyzing the expected evolution of $\lambda$ and $L_{s c}$ for increasing values of the number of structural sections $n_{L S}$ where a given mechanism occurs (which can be seen as a proxy for the behavior of the building), $\lambda$ and $L_{s c}$ are expected to have opposite evolution trends similar to those represented in the qualitative graph of Fig. 1 . When $n_{L S}$ increases, $\lambda$ is seen to decrease, since higher intensity ground motions (with lower probability of occurrence) are required to reach the limit state capacity at a larger number of sections. On the 


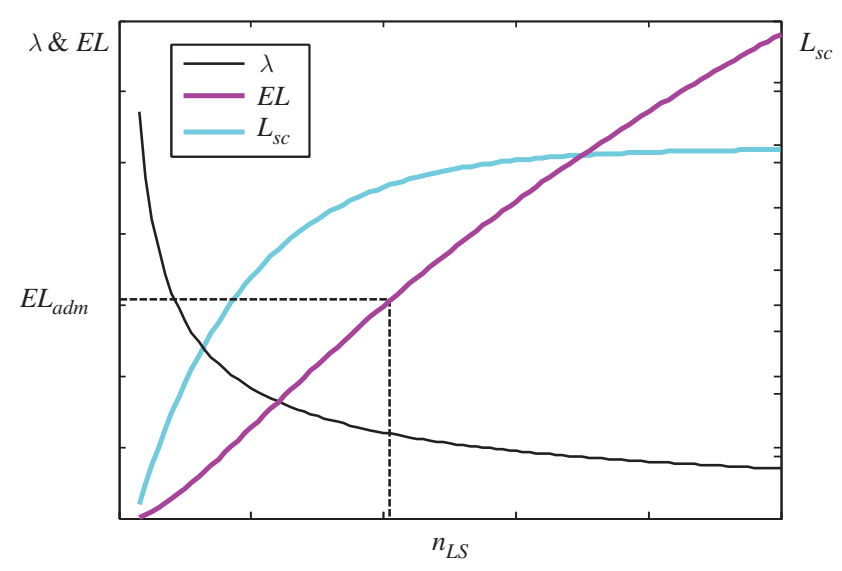

FIGURE 1 Expected qualitative evolution of $\lambda, L_{s c}$, and $E L$ for increasing values of $n_{L S}$. (C) Nova Science Publishers, Inc. Reproduced by permission of Nova Science Publishers, Inc. Permission to reuse must be obtained from the rightsholder.

other hand, the value of $L_{s c}$ increases since admitting that a larger number of sections can reach the EDP capacity also leads to higher levels of global building damage. Figure 1 also shows the evolution of $E L$ which increases as $n_{L S}$ increases. Based on the value set for $E L_{a d m}$, the admissible building performance corresponds to the largest value of $n_{L S}$ that conforms with $E L_{a d m}$.

\subsection{Estimating the Limit State Fragility Curve by the IM-based Approach}

The quantification of $\lambda$ for a given limit state according to Eq. (3) requires an earthquake hazard curve in terms of the selected IM and the fragility curve $p_{f}$ of that limit state. The determination of $p_{f}$ involves the characterization of the structural behavior, usually obtained by the numerical simulation of a detailed mathematical model of the structure subjected to sets of earthquake ground motions scaled for increasing intensities. As previously referred, the fragility curve is estimated by the IM-based approach [Ibarra et al., 2002], which is an efficient procedure that makes use of the IM-capacity concept and can be dated back to the works of Veneziano et al. [1983]. The IM-based approach defines the fragility curve using a random variable, termed the IM-capacity $\left(I M_{C}\right)$, that represents the ground motion intensity at which a given limit state occurs for the structure under assessment. Several realizations of $I M_{C}$ associated to the selected limit state can then be obtained by analyzing the structure under a set of earthquake records using the IDA procedure [Vamvatsikos and Cornell, 2002] where each record is scaled for increasing intensities until the limit state occurs. The cumulative distribution function (cdf) defined by the statistical distribution of the several $I M_{C}$ realizations represents the fragility curve of the selected limit state. This fragility curve has been, in many cases [Ibarra and Krawinkler, 2005; Jalayer et al., 2007; Zareian and Krawinkler, 2007; Bradley and Dhakal, 2008; Liel et al., 2009; Haselton et al., 2011; Liel et al., 2011] assumed to be well represented by a lognormal cdf which enables it to be written as:

$$
p_{f}(i m)=P\left(I M_{C} \leq I M=i m\right)=\Phi\left(\frac{\ln i m-\ln \eta_{I M_{C}}}{\beta_{I M_{C}}}\right),
$$


where $\Phi($.$) is the normal cdf, and \ln \eta_{I M_{C}}$ and $\beta_{I M_{C}}$ are the mean and the standard deviation, respectively, of the distribution. In this approach, the value of $\beta_{I M_{C}}$ represents the uncertainty in the estimate of $I M_{C}$ due to several factors, e.g., due to the record-to-record variability of the demand, among others (e.g. see ATC, 2009).

\subsection{Estimating the Limit State Expected Loss $L_{s c}$}

The expected value of the loss $L_{s c}$ associated to the occurrence of a given limit state is estimated using the storey-based approach proposed by Ramirez and Miranda [2009]. This approach has established loss curves representing the losses of all the individual components of an entire building storey as a function of a selected EDP, $L_{s c \mid E D P}$. Different curves have been defined to quantify the losses in structural and non-structural components, and different EDPs were also selected depending on the type of component. To illustrate this type of data, Figure 2 presents $L_{s c \mid E D P}$ loss curves for structural and interstorey drift-sensitive non structural components associated to different stories of a mid-rise reinforced concrete interior frame of an office building (based on the data presented by Ramirez and Miranda, 2009). For both cases, the selected EDP was the inter-storey drift $\Delta$. The presented loss values, which are normalized by the storey replacement cost, can be seen to amount to maximum values from $20-25 \%$ of the story cost, for the case of the structural losses, and between $40-50 \%$ of the story cost, for those associated to the inter-story drift-sensitive non structural components. The remaining losses are assigned to acceleration-sensitive non structural components [Ramirez and Miranda, 2009].

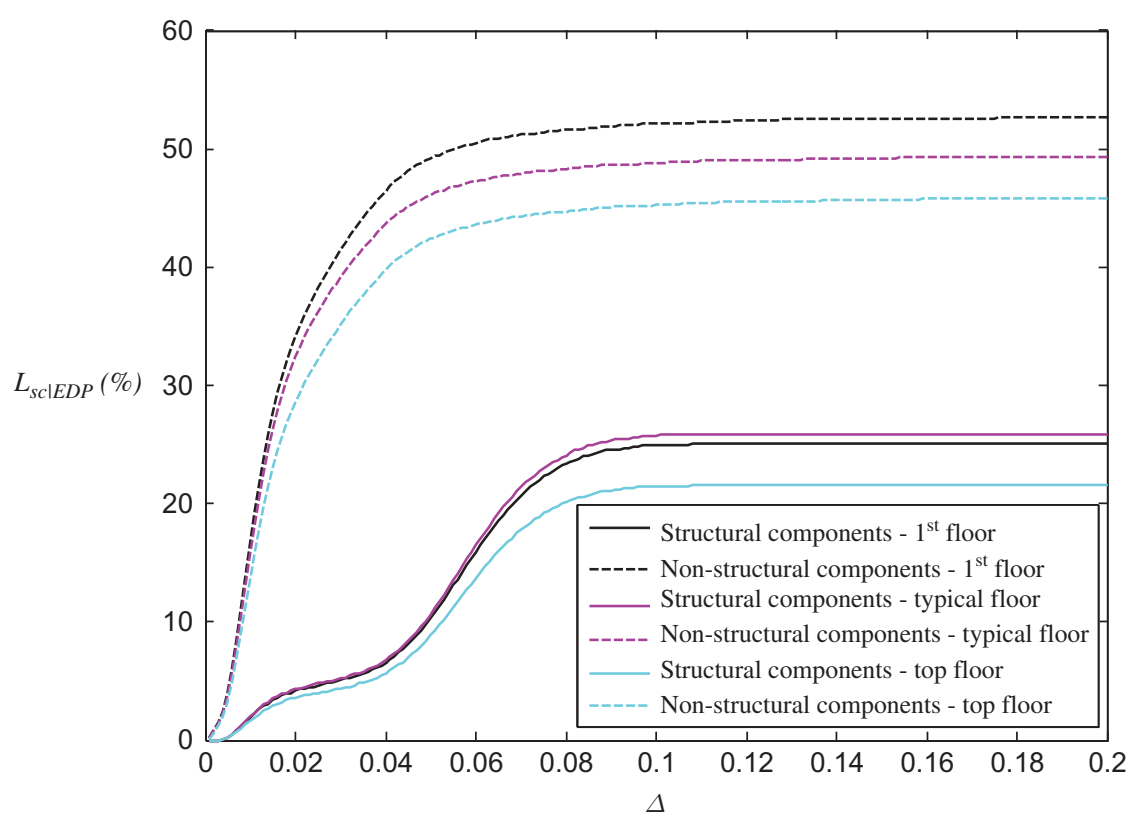

FIGURE 2 Loss curves for structural and inter-story, drift-sensitive, non structural components associated for a mid-rise reinforced concrete interior frame of an office building (based on the data presented by Ramirez and Miranda, 2009). (C) Nova Science Publishers, Inc. Reproduced by permission of Nova Science Publishers, Inc. Permission to reuse must be obtained from the rightsholder. 
To quantify the expected loss value associated to the ith building story, $L_{s c, i}$, due to the occurrence of a given limit state, the $L_{s c \mid E D P, i}$ curve must be combined with the exceedance probability of the selected EDP at the ith story, $P\left(E D P_{i}>e d p_{i}\right)$. The probabilistic characterization of the $i$ th story EDP can be defined by determining the EDP values corresponding to the several $I M_{C}$ realizations, $E D P_{C}$. The cdf of these $E D P_{C}$ values represents the fragility curve of the ith story EDP associated to the occurrence of the limit state under analysis, $p_{E D P_{C, i}}$, which can also be assumed to be represented by a lognormal cdf:

$$
p_{E D P_{C, i}}(e d p)=P\left(E D P_{C, i} \leq E D P=e d p\right)=\Phi\left(\frac{\ln e d p-\ln \eta_{E D P_{C, i}}}{\beta_{E D P_{C, i}}}\right),
$$

where $\ln \eta_{E D P_{C, i}}$ and $\beta_{E D P_{C, i}}$ are the mean and the standard deviation, respectively, of the EDP distribution at the ith story. The value of $L_{s c, i}$ can then be obtained by:

$$
\begin{aligned}
L_{s c, i} & =\int_{0}^{\infty} E\left(L_{s c, i} \mid E D P_{C, i}\right) \cdot d P\left(E D P_{C, i}>e d p_{i}\right) \\
& =\int_{0}^{\infty} L_{s c \mid E D P_{C, i}}(x) \cdot\left|\frac{d}{d x} \Phi\left(\frac{\ln x-\ln \eta_{E D P_{C, i}}}{\beta_{E D P_{C, i}}}\right)\right| d x
\end{aligned}
$$

in which $L_{s c \mid E D P_{C, i}}$ represents $L_{s c \mid E D P, i}$ for the case where the EDP values correspond to those of $E D P_{C}$. Finally, the total expected value of the loss $L_{s c}$ associated to the occurrence of the limit state under analysis is obtained by summing the losses of each story.

By using this approach, the proposed methodology assumes that the expected losses of each storey are independent from each other. However, there are situations where the loss in a component of a given story is also dependent on the behavior of another story. Although the proposed methodology does not capture losses in building components that are dependent between stories, a procedure to modify the loss functions in order to account for this dependency is discussed by Ramirez and Miranda [2009].

\subsection{Definition of the Limit States}

The proposed methodology involves the probabilistic performance analysis of structures considering code-defined limit states which are usually associated to damage control, life safety or near collapse conditions. Although the considered limit state definitions are based on those presented in EC8-3 [CEN, 2005], the current proposals can be related to other international standards that involve similar limit states or performance levels.

The performance requirements associated to the limit states defined by EC8-3 are described in qualitative terms and make reference to different damage states which are related to the global behavior of the structure. Three damage states of increasing severity are established by EC8-3 and the corresponding limit states are termed: Damage Limitation (DL), Significant Damage (SD), and Near Collapse (NC). Although defined in terms of the global (system-level) behavior of the structure, the safety verifications required to meet these limit states are established using parameters associated to the behavior of structural members (i.e. using a local level approach). According to EC8-3, as well as other international standards, if the member mechanism associated to a given limit state is ductile, the general approach is to check if the member deformation demand is lower than an admissible deformation capacity. In case the member mechanism associated to a given limit state 
is of the brittle type, one has to check if the member capacity in terms of strength is not exceeded by the corresponding demand. The safety of a given mechanism associated to a certain limit state can then be accepted when the damage variable $\rho$ associated to the member verifies the condition

$$
\rho=\frac{D}{C} \leq 1
$$

where $D$ is the member mechanism demand and $C$ is the limit state capacity of the mechanism. From the point of view of the global safety of the structure, a literal interpretation of the EC8-3 procedures appears to indicate that compliance with a selected limit state is only accepted when all the individual structural members/sections meet their corresponding safety requirement. As discussed next, this condition is found to be insufficient. Given the format of the methodology presented herein, an update of the limit state definitions is proposed.

2.4.1. The Limit State of Damage Limitation. According to EC8-3, a structural member conforms to the DL limit state when its deformation does not exceeds the yield chord rotation. Furthermore, if demand is found to exceed the yield deformation in only one member of the building, irrespective of which member, the whole building is considered to be nonconforming. Based on this interpretation, one can argue that such condition is not easily correlated with a global limit state description that requires "the structure to be only lightly damaged, with structural elements prevented from significant yielding and retaining their strength and stiffness properties" [CEN, 2005] and that indicates the possibility of having more than one yielding section.

A conformity condition based on risk and loss criteria is therefore proposed instead. This condition accepts the occurrence of the limit state in several scenarios, as long as the corresponding value of $E L$ is not greater than an admissible value $E L_{a d m, D L}$. In this case, each scenario corresponds to a different number $n_{D L}$ of structural sections reaching or exceeding the yield limit. By defining the admissible consequences of reaching this limit state in terms of the $E L_{a d m, D L}$, which is a function of $\lambda_{a d m, D L}$ and $L_{s c ~ a d m, D L}$, the number of admissible yielding sections is set by a more rational decision-making process. Since the global performance associated to the DL limit state is defined by a low level of structural damage, it is suggested that $L_{s c ~ a d m, D L}$ should reflect repair costs due to non structural damage only.

2.4.2. The Limit State of Significant Damage. When analyzing the limit state of SD, a scenario for which the code interpretation of the limit state is insufficient can also be identified. When comparing the situation where there is only one section with a $\rho$ value above 1.0, irrespective of the $\rho<1$ values of the remaining sections (which includes the possibility of having higher values closer to 1 or a scenario where most values are low), with the situation where there is a significant number of sections with $\rho$ values in the range $0.85-0.95$ but none above 1 , the code procedure implies the latter case to be conforming to the limit state, while the former is not. Based on the description of the SD limit state proposed by EC8-3, which refers, among other aspects, that "the structure will be significantly damaged and likely to be uneconomic to repair" [CEN, 2005], one can argue that the high $\rho$ values of the second case involve a level of damage that could be higher than that of the first. Therefore, from an economic point of view, the higher level of loss involved in the second case may not be adequately captured by the code provisions. 
The focus of the revised definition addresses the number of structural sections where the deformation limit can be attained, as in the previous limit state, but also the level of deformation defined for such limit value. Therefore, a conformity condition based on risk and loss criteria is also proposed which establishes that the occurrence of the limit state is accepted in several scenarios, as long as the corresponding value of $E L$ is not greater than an admissible value $E L_{a d m, S D}$. In this case, each scenario corresponds to a different number $n_{S D}$ of structural sections reaching or exceeding a selected deformation limit $d_{\text {lim }}$. It is believed that $d_{\text {lim }}$ should be defined based on economic considerations involving estimates of the expected damage-related costs. The value of $E L_{a d m, S D}$ is a function of $\lambda_{a d m, S D}$ and $L_{s c \text { adm,SD, }}$, where the latter should reflect the maximum admissible cost for the repair of the whole structure. Therefore, $L_{s c}$ adm,SD should reflect the admissible value of the repair costs of both the structural and the non structural elements.

2.4.3. The Limit State of Near Collapse. With respect to the limit state of NC, EC83 refers, among other aspects, that "the structure will be heavily damaged, with low residual lateral strength and stiffness" [CEN, 2005]. Since the structure is expected to remain standing after a severe earthquake, the NC limit refers to a state of the structure that precedes global collapse. However, it is believed that its level of damage renders the building uneconomic to repair. For this limit state, the EC8-3 verification procedure only accounts for failure prevention at the section level and does not contemplate the potential development of global yield mechanisms. Following the definition proposed in fib [2003a] and Jalayer et al. [2007], the development of a yield mechanism (or plastic mechanism) corresponds to a situation involving the yielding of several sections (with different levels of inelastic demand) that forms a structural configuration having a significantly reduced lateral stiffness. Although this type of global structural behavior fits in the general description of the NC limit state of EC8-3, the corresponding safety verifications do not contemplate such mechanisms. With respect to member failure prevention, EC8-3 makes no distinction between column and beam failure. As generally accepted (e.g., see Pinto et al., 2004; Deierlein and Haselton, 2005; Jalayer et al., 2007; Fajfar and Dolšek, 2012), a column failure (either by reaching its deformation or shear force capacity) is more severe than a beam failure.

The revised definition of this limit state involves bounding conditions different than those of the previous limit states. Given that, when reaching this limit state, the building will not be repairable, a bounding condition in terms of admissible loss is not considered relevant. Hence, the building performance is controlled by limiting $\lambda$ to an admissible value $\lambda_{a d m, N C}$, and by defining conditions in terms of the number of sections where a given demand/mechanism is accepted. With respect to the latter, distinction must be made between mechanisms occurring in beams and in columns. Given the larger severity of the consequences due to the failure of a column, the occurrence of the NC limit state at a single section is considered to be enough to reflect a nonconforming structure. On the other hand, for beams, it is considered that the limit state capacity of the considered mechanism can occur at several sections. In this case, a non conforming condition is established when the $\mathrm{NC}$ limit state occurs in all the beam sections of a given story.

In addition to the local (section level) analysis of the demand, a global analysis of the building behavior is also carried out in order to include the occurrence of the previously referred global yield mechanisms. Existing methodologies identifying the occurrence of such yield mechanisms can be divided in two main types: methodologies tracking the evolution, for increasing levels of the selected IM, of a demand parameter capable of reproducing the global behavior of the building [Vamvatsikos and Cornell, 2002] or approaches 
tracking the occurrence of a number of yield mechanisms specified beforehand [Jalayer et al., 2007].

The first type of methodologies is mostly associated to procedures aiming to track the occurrence of global structural collapse such as, for example, the ATC-63 framework [ATC, 2009]. Furthermore, the accuracy of this approach depends on the adequacy of the relation (i.e., the IDA curve) between the selected EDP (usually the inter-story drift) and the IM to represent the occurrence of these mechanisms. The development of these mechanisms is typically associated to a flatness of the IDA curve representing a very large increase of the EDP for a small increase of the IM which, in turn, reflects the considerable reduction of the global stiffness of the structure, e.g., such as when a soft-story mechanism develops. If the referred flatness does not occur, the identification of a global mechanism becomes more difficult and may only be possible if deterioration is modelled in the structural analysis [Krawinkler and Lignos, 2009]. With respect to the second approach, although the procedure proposed by Jalayer et al. [2007] has the potential to consider any type of global mechanism, it has the downside of requiring them to be identified before the analysis. Given the multitude of possibilities, the adequacy of this approach relies considerably on the opinion and experience of the analyst. Furthermore, this procedure also disregards the realistic correlation between the behavior of the components that are part of the mechanism since it considers that a mechanism occurs when its strongest component begins to yield [Jalayer et al., 2007], i.e., a mechanism is assumed to be formed by a parallel arrangement of fully correlated components. To overcome the disadvantages of both approaches, an alternative methodology is proposed which tracks the occurrence of any type of yield mechanism without the need to identify them before the analysis.

The proposed approach identifies the occurrence of yield mechanisms by assessing the singularity of an equivalent stiffness matrix representing the current state of the building behavior. This approach is defined by the following steps which are carried out at each time increment of the nonlinear dynamic analysis.

Step 1. Check the behavior state of each structural section to determine if its current loading state is located in a positive or negative post-yield loading branch of the behavior path. Sections meeting this condition are termed active yielding sections. To illustrate the definition of the referred loading branches, Fig. 3 presents a generalized forcedisplacement relation representing different stages of the hysteretic behavior. In Fig. 3, branches 3 and 10 are positive post-yield loading branches, the latter occurring after a negative loading cycle and a positive reloading, while branch 6 is a negative post-yield loading branch.

Step 2. If one or more active yielding sections are found, an equivalent elastic EulerBernoulli stiffness matrix of the structure $K_{e q}$ is formulated with zero-stiffness terms assigned to the flexural terms of those sections.

Step 3. If $K_{e q}$ is singular, the structure is unstable [Nafday, 2008], the corresponding configuration of active yielding sections is that of a yield mechanism, and the corresponding IM value of the ground motion is recorded.

By using such procedure, it is possible to identify any type of yield mechanism taking into account the correlation between the behavior of the components forming the mechanism and accounting only for sections actively loaded with post-yield stiffness. Furthermore, to account for the case where sections might possess a post-yield stiffness that still offers a relevant contribution against collapse, such sections might be required to reach a certain level of ductility demand before terming them active yielding sections. This additional condition can also be analyzed when checking the section's loading state in Step 1. 


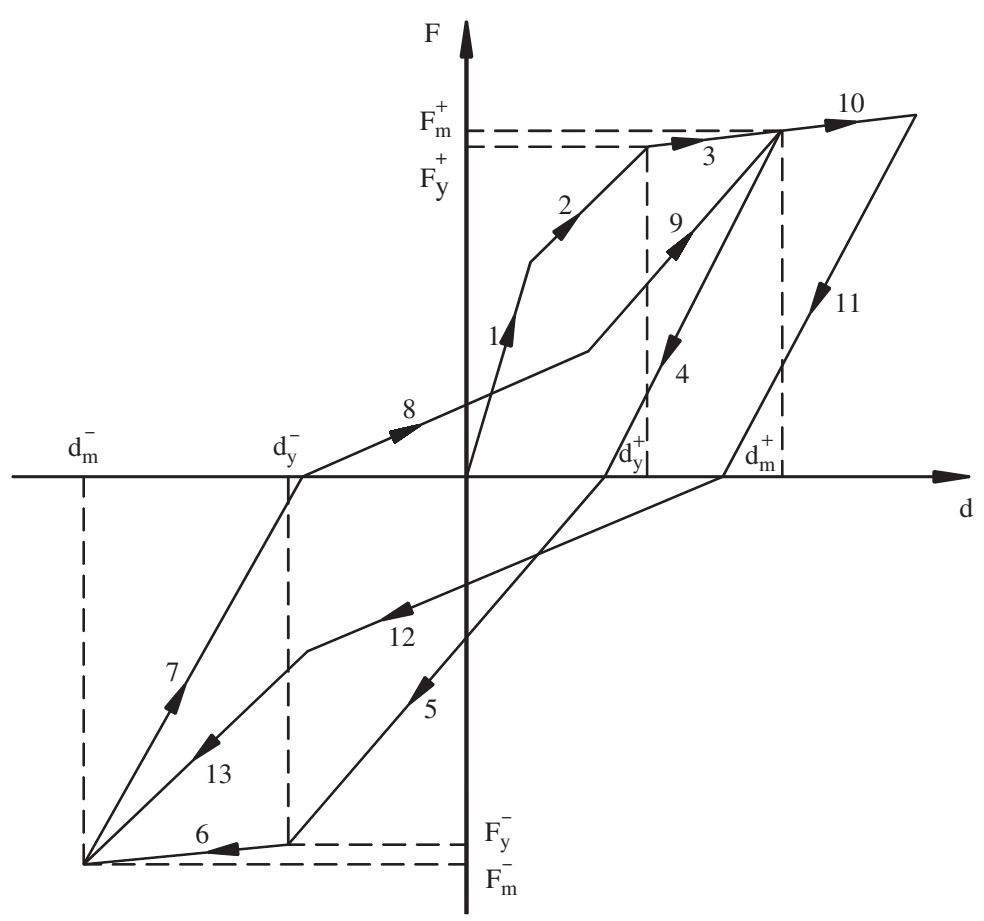

FIGURE 3 Generalized hysteretic force-displacement relation. (C) Nova Science Publishers, Inc. Reproduced by permission of Nova Science Publishers, Inc. Permission to reuse must be obtained from the rightsholder.

\subsection{Accounting for the Uncertainty in the Limit State Capacities}

As referred by Jalayer et al. [2007], the uncertainty associated to the modelling of member limit state capacities has a significant contribution to $\lambda$. Depending on the considered standard, the limit state capacities are seen to be established either by fixed values of a given EDP (e.g., see ASCE, 2007) or by semi-empirical expressions (e.g., see CEN, 2005) defining the limit values of the referred EDPs. In the former case, the proposed values are expected to be conservative estimates of the real capacity [Haselton, 2006] while in the latter, the average estimates provided by the referred expressions are known to have a large uncertainty [Haselton, 2006; fib, 2003a,b). In this case, the uncertainty in the limit state capacities can be associated to the modelling error deriving from the proposed capacity formulas as well as to the variability of the mechanical parameters entering those formulas [Jalayer et al., 2007].

Among the different methods which are available to account for this uncertainty (e.g., see Pinto et al., 2004; Jalayer et al., 2007; Liel et al., 2009), the selected approach assumes that limit state capacities $C$ can be modeled according to the following general format:

$$
C=\hat{C} \cdot \varepsilon_{U C}
$$

where $\hat{C}$ is the estimate given by the referred semi-empirical expressions, and $\varepsilon_{U C}$ is a lognormal random variable with unit median and a dispersion $\beta_{U C}$ accounting for the variability sources previously mentioned. In order to reflect the section-level limit state capacity uncertainty at the system level, i.e., in the uncertainty associated to the estimate of $I M_{C}$, the 
correlation between the capacities of different sections must be accounted for. To address this issue, an approach similar to the one proposed by Jalayer et al. [2007] is considered. Therefore, it is assumed that for a given mechanism (e.g., yield deformation, shear failure) the limit state capacities of all members are fully correlated. On the other hand, for a given member, the limit state capacities of different mechanisms are considered to be uncorrelated. Given this assumption, the effect of this uncertainty on the estimate of $I M_{C}$ can be included by sampling different realizations of the individual member capacities using Eq. (8) which are then paired with the IDA curves obtained from the considered earthquake ground motions. Therefore, for a given IDA curve, an array of member capacities (i.e., a number of realizations, $n_{U C}$, of the capacities for each member) is established and each sample of capacities (i.e., one realization of the capacity of each member) will lead to a different realization of the $I M_{C}$ associated to the limit state under analysis. Using this approach, the quantification of parameters $\eta_{I M_{C}}$ and $\beta_{I M_{C}}$ which characterize the limit state fragility curve, Eq. (4), can account for the uncertainty in the member capacities.

It is further noted that, for simplicity, the effect of the uncertainty in the limit state capacities was not incorporated in the prediction of structural demand. Nevertheless, if such component of the variability of the structural demand is wanted, procedures such as those proposed by Liel et al. [2009] or Vamvatsikos and Fragiadakis [2010] can be used.

\subsection{Stepwise Description of the Proposed Methodology}

Based on the individual features addressed over the previous sections, the sequence of steps involved in the presented probabilistic methodology for the analysis of building performance is described in the following.

The proposed method assumes that a set of $n_{g m}$ IDA curves with an adequate number of IM levels have been obtained from the analysis of the structure subjected to $n_{g m}$ ground motion records scaled to those IM levels. After selecting the limit state for which the performance of the structure is to be assessed, the following steps must be carried out.

Step 1. For a given limit state, select the type of section level mechanism for which seismic safety is going to be analyzed (e.g., a deformation or a force-based mechanism).

Step 2. Define a value for $n_{U C}$ (the number of realizations of the capacity of each section) and sample $n_{U C}$ values of $\varepsilon_{U C}$ from its distribution.

Step 3. Select a value for $n_{L S}$ (the number of structural sections where the limit state mechanism is allowed to occur).

Step 4. Select a value of $\varepsilon_{U C}$ from those sampled in Step 2.

Step 5. Select one IDA curve from the set of $n_{g m}$ curves.

Step 6. Select the first IM level from the current IDA curve.

Step 7. Determine $\rho=\frac{D}{\hat{C} \cdot \varepsilon_{U C}}$ for all the $n_{\text {sec }}$ sections of the structure.

Step 8. Determine the number of sections $n_{\rho>1}$ with $\rho$ values larger than 1.0.

Step 9. If $n_{\rho>1}<n_{L S}$, select the next IM level and repeat the procedure from Step 7; if $n_{\rho>1} \geq n_{L S}$, record the current IM level, which corresponds to a realization of $I M_{C}$ (the ground motion intensity at which the limit state scenario occurs), e.g., see Fig. 4a, and proceed to the next IDA curve to repeat the procedure from Step 6.

Step 10. After going through all the IDA curves, the procedure is repeated from Step 5 for a different value of $\varepsilon_{U C}$, until the whole $n_{U C}$ values have been considered.

Step 11. Characterize the limit state fragility curve by Eq. (4) using the $I M_{C}$ realizations, e.g., see Fig. $4 b$.

Step 12. Determine $\lambda$ by Eq. (3). 

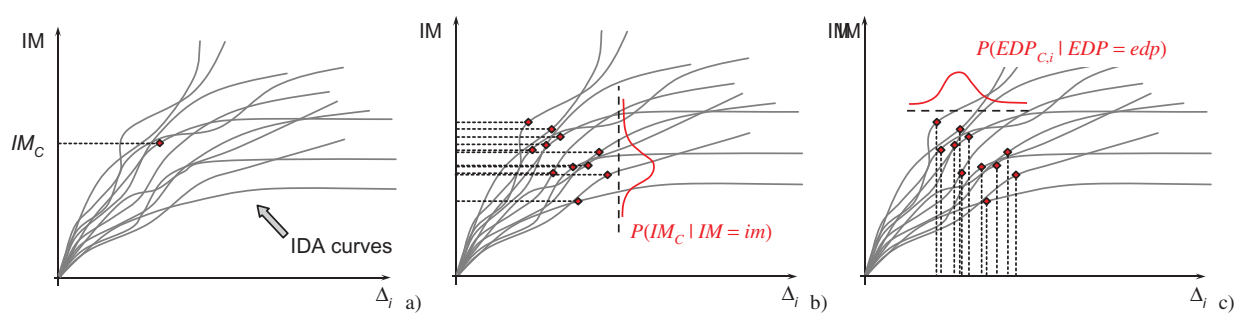

FIGURE 4 Illustration of Step 9: (a) of step 11 considering $n_{U C}=1$ (b) and of Step 13 considering $n_{U C}=1$ and for the ith story (c). (C) Nova Science Publishers, Inc. Reproduced by permission of Nova Science Publishers, Inc. Permission to reuse must be obtained from the rightsholder.

Step 13. Characterize the fragility curves of the selected EDP of each storey for the storybased loss quantification by Eq. (5), e.g., see Fig. 4c.

Step 14. Determine the expected value of the loss of each story by Eq. (6).

Step 15. Determine $L_{s c}$ for the current limit state scenario.

After these steps, the triplet $\left(n_{L S} ; \lambda ; L_{s c}\right)$ defines a limit state performance scenario. The building performance quantification procedure is then repeated from Step 4 for a different value of $n_{L S}$. In order to obtain an adequate representation of the building performance evolution for different $n_{L S}$ values, it is suggested that the analysis starts by setting $n_{L S}$ equal to one and that subsequent repetitions of the procedure increase it by single units. The several performance triplets are then combined to obtain $E L$ according to Eq. (1). The value of $E L$ is then analyzed in light of the limit defined by $E L_{a d m}$ to determine which combination of scenarios is admissible for the current limit state.

In a situation where the uncertainty of the member limit state capacities is not considered, the procedure is simplified by considering both $n_{U C}$ and $\varepsilon_{U C}$ equal to 1.0 in the necessary steps. According to the conditions defined in Sec. 2.4.3 for the limit state of NC, Steps 13,14, and 15 do not apply for this limit state since the building performance is only controlled by $\lambda$. Furthermore, when checking for the occurrence of yield mechanisms, Step 10 needs to include the tracking procedure presented in Sec. 2.4.3.

\section{Example Application of the Proposed Methodology}

An application of the proposed methodology is presented in the following addressing the seismic performance assessment of a RC structure for the previously defined limit states. A general overview of the structure, of the component capacities and their corresponding probabilistic modelling, of the selected hazard scenario, of the cost analysis data and of the selected performance conditions is briefly presented. Details about the numerical modelling of the structure and the selected ground motions defining the seismic demand are omitted for the sake of brevity and can be found in Romão et al. [2011, 2012a].

\subsection{General Description of the Selected Structure}

The selected structure is the six-storey irregular RC frame presented by Ferracuti et al. [2009]. The elevation view of the frame, referred herein as IRREG6, is presented in Fig. 5 along with the column cross section dimensions. All the beams are $0.30 \times 0.50 \mathrm{~m}^{2}$. A steel 


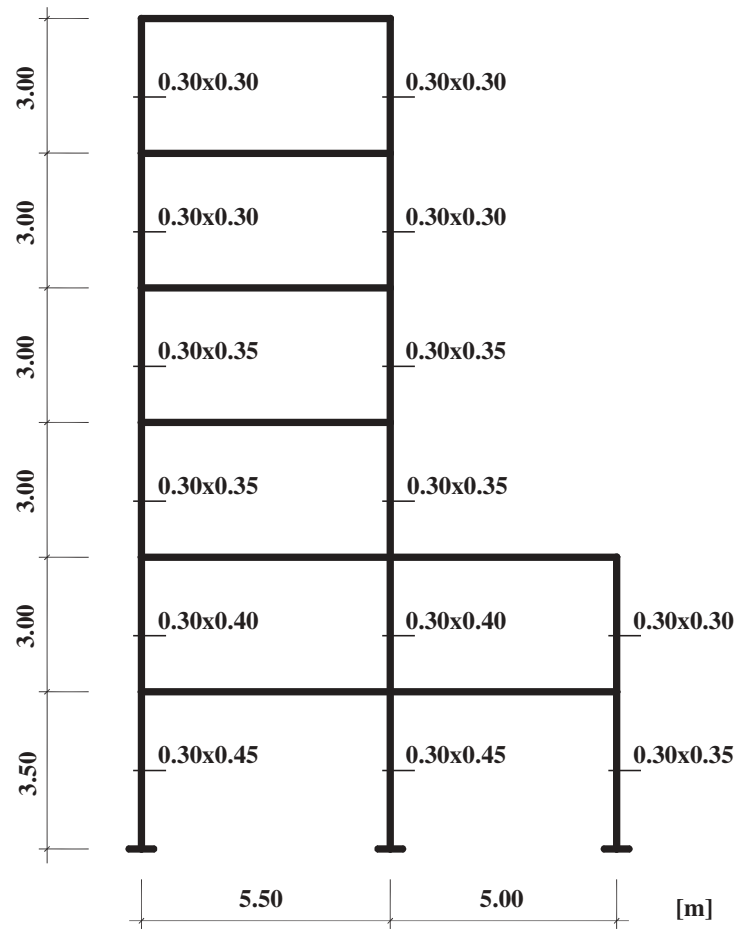

FIGURE 5 Elevation view of the IRREG6 frame and its column cross section dimensions. (C) Nova Science Publishers, Inc. Reproduced by permission of Nova Science Publishers, Inc. Permission to reuse must be obtained from the rightsholder.

with a yield strength of $414 \mathrm{MPa}$ and a concrete with a compressive strength of $33 \mathrm{MPa}$ were considered, following the values proposed by Ferracuti et al. [2009] for the material properties. Additional information concerning the frame characteristics can be found in Ferracuti et al. [2009].

\subsection{Component Capacities and Probabilistic Modeling of Their Uncertainty}

Component capacities were defined according to the models proposed by EC8-3 [CEN, 2005]. Ductile capacities are defined in terms of the admissible DL, SD, and NC member chord rotations, while brittle capacities are characterized by the admissible NC shear force. For the quantification of the DL chord rotation capacity $\theta_{D L}$, and assuming that no shear cracking is expected to precede flexural yielding, EC8-3 proposes the following expression

$$
\theta_{D L}=\phi_{y} \cdot \frac{L_{s}}{3}+0.0013 \cdot\left(1+1.5 \frac{h}{L_{s}}\right)+0.13 \cdot \phi_{y} \cdot \frac{d_{b} \cdot f_{y}}{\sqrt{f_{c}}}
$$

in which $\Phi_{y}$ is the yield curvature of the member end section, $L_{s}$ is the shear span, $h$ is the cross section depth, $d_{b}$ is the mean diameter of the tension reinforcement, $f_{y}$ is the longitudinal reinforcement yield strength, and $f_{c}$ is the concrete compressive strength.

In order to account for the uncertainty in $\theta_{D L}$ according to Eq. (8), $50 \varepsilon_{U C}$ values are sampled from its distribution, where the dispersion $\beta_{U C}$ for $\theta_{D L}, \beta_{U C, \theta_{D L}}$, is considered to 
be 0.36 [fib, 2003a] in order to reflect the uncertainty due to the modelling error of Eq. (9). The uncertainty due to the variability of the mechanical parameters entering Eq. (9) is not taken into account since its effect is considered to be negligible when compared to that of the modeling error.

For the quantification of the NC chord rotation capacity $\theta_{N C}$, the following semiempirical expression proposed by EC8-3 is considered herein:

$$
\theta_{N C}=\frac{1}{\gamma_{e l}}\left[\theta_{D L}+\left(\phi_{u}-\phi_{y}\right) \cdot L_{p l} \cdot\left(1-\frac{L_{p l}}{2 L_{s}}\right)\right],
$$

where $\Phi_{u}$ is the ultimate curvature of the member end section and $L_{p l}$ is the plastic hinge length. The definition of $\Phi_{u}$ and of $L_{p l}$ depends on the selected confined concrete model [CEN, 2005]. The purpose of factor $\gamma_{e l}$ is to account for the variability of the capacity. In the present application, $\gamma_{e l}$ is considered equal to one since the variability of the capacity values is simulated. To account for the uncertainty in $\theta_{N C}$ according to Eq. (8), $50 \varepsilon_{U C}$ values are sampled from its distribution, where the dispersion $\beta_{U C}$ for $\theta_{N C}, \beta_{U C, \theta_{N C}}$, is considered to be 0.90 [ $f i b, 2003 \mathrm{a}]$ in order to reflect the uncertainty due to the modelling error of Eq. (10). As for the case of $\theta_{D L}$, the uncertainty due to the variability of the mechanical parameters is also not taken into account.

For the case of the SD limit state, EC8-3 states that the corresponding chord rotation capacity $\theta_{S D}$ is defined as $\theta_{S D, 1}=0.75 \theta_{N C}$. In addition to this case, the performance analysis of the structure for this limit state was also carried out for the situation where a chord rotation capacity $\theta_{S D, 2}=0.85 \theta_{S D, 1}$ is considered. This additional analysis is performed in order to address the considerations discussed in Sec. 2.4.2. Since $\theta_{S D}$ is a function of $\theta_{N C}$, the uncertainty in $\theta_{S D}$ is that of $\theta_{N C}$.

With respect to the shear force capacity $V_{N C}$ for the limit state of NC, EC8-3 proposes the formulation defined by:

$$
\begin{aligned}
& V_{N C}=\frac{1}{\gamma_{e l}}\left[\frac{h-x}{2 \cdot L_{s}} \cdot \min \left(N ; 0.55 \cdot A_{c} \cdot f_{c}\right)+\left(1+0.05 \cdot \min \left(5 ; \mu_{\Delta}^{p l}\right)\right) .\right. \\
& \left.\cdot\left[0.16 \cdot \max \left(0.5 ; 100 \cdot \rho_{t o t}\right) \cdot\left(1-0.16 \cdot \min \left(5 ; \frac{L_{s}}{h}\right)\right) \cdot \sqrt{f_{c}} \cdot A_{c}+V_{w}\right]\right]
\end{aligned}
$$

where $x$ is the compression zone depth, $N$ is the compressive axial force (equal to zero for tension), $A_{c}$ is the cross section area taken equal to $b_{w} \cdot d$ ( $b_{w}$ is the section width and $d$ is the structural depth), $\rho_{\text {tot }}$ is the total longitudinal reinforcement ratio, $\mu_{\Delta}^{p l}$ is the ratio between the plastic part of the chord rotation demand and the yield chord rotation given by Eq. (9), and $V_{w}$ is the contribution of transverse reinforcement to shear resistance [CEN, 2005]. To account for the uncertainty in $V_{N C}$ according to Eq. (8), $50 \varepsilon_{U C}$ values are sampled from its distribution, where the dispersion $\beta_{U C}$ for $V_{N C}, \beta_{U C, V_{N C}}$, is considered to be 0.14 [ $f i b$, 2003 b] in order to reflect the uncertainty due to the modeling error of Eq. (11).

\subsection{Seismic Demand and Hazard Scenario}

The seismic demand considered for each structure consisted of a suite of 50 real ground motions extracted from the Pacific Earthquake Engineering Research Center NGA database [PEER-NGA, 2009] according to the criteria defined in Romão et al. [2011, 2012a]. The structure was analyzed using incremental dynamic analysis (IDA) [Vamvatsikos and Cornell, 2002] where the selected ground motions are scaled for increasing values of $S_{a}\left(T_{1}\right)$ 
until the selected limit state is attained $-S_{a}\left(T_{1}\right)$ is the $5 \%$ damping spectral acceleration ordinate of the ground motion for the fundamental period of the structure $T_{1}$ which is the selected intensity measure (IM) and is simply referred to as $S_{a}$ hereon.

In order to define the earthquake hazard curve $H(x)$ required for the methodology, seismic hazard data was obtained for the $S_{a}$ values of the structure, and for a reference period of one year. The selected methodology and data are those considered to carry out the hazard studies supporting the seismic zonation and the seismic action levels of the Portuguese National Annex of Eurocode 8 [EC8-1, 2009], e.g., see the probabilistic seismic hazard analysis presented by Campos Costa et al. [2008]. The considered seismic hazard was defined for the intraplate seismic action and a soil of Type B [EC8-1, 2009], referring to events with their epicentres mainly inland, in which the model of mainland gross-source zones and the parameters defining the seismic occurrence process in each source zone, such as the Poissonian process and the exponential distribution of magnitudes, were adapted from the proposals presented by Sousa and Oliveira [1996]. The attenuation of intensity with distance was described by the relationships defined by Ambraseys et al. [1996].

\subsection{Cost Analysis Data and Performance Conditions}

The expected loss value associated to the $i t h$ building story, $L_{s c, i}$, is quantified using the $L_{S C \mid E D P}$ structural and non structural loss curves for mid-rise RC interior frames of an office building defined by Ramirez and Miranda [2009] as a function of a selected EDP. In order to simplify the proposed example applications, only non structural losses associated to interstory drift-sensitive non structural components are considered herein. The considered loss curves are presented in Fig. 2.

With respect to the selected values of the admissible expected losses $E L_{a d m}$ defined by Eq. (2), values for $\lambda_{a d m}$ and $L_{s c ~ a d m}$ were set for the considered limit states. For the case of $\lambda_{a d m}$, it is referred that, for existing structures, current standards and/or available technical documents on the subject do not have definitive proposals on this matter. Therefore, the $\lambda_{\text {adm }}$ values considered herein were defined as a reduction of the target reliability values for new structures proposed by JCSS [2001] for a one year reference period and for ultimate limit states. As referred by Diamantidis and Bazzurro [2007], this approach assumes that achieving a higher reliability level in existing structures has a higher cost when compared to that of structures under design. The $\lambda_{\text {adm }}$ values considered for the selected limit states are presented in Table 1 and are based on those proposed by JCSS [2001] for the higher category of the relative cost of implementing safety measures. These $\lambda_{\text {adm }}$ values are defined for the reference period of one year and were associated to small, moderate, and large risks to life and economic consequences for the limit states of DL, SD and NC, respectively. Along with the values for $\lambda_{a d m}$, Table 1 also presents the corresponding reliability indexes $\beta$.

With respect to $L_{s c ~ a d m}$, the considered limit values correspond to average repair costs of all the building stories. A value of $10 \%$ was assumed for the DL limit state (considering

TABLE 1 Considered values for $\lambda_{a d m}$ for the selected limit states and the corresponding reliability indexes $\beta$

\begin{tabular}{lcc}
\hline Limit state & $\lambda_{a d m}$ & $\beta$ \\
\hline DL & 0.001 & 3.09 \\
SD & 0.0002 & 3.54 \\
NC & 0.0001 & 3.70 \\
\hline
\end{tabular}


only losses associated to inter-story drift-sensitive non structural components) and a value of $25 \%$ was assumed for the SD limit state (considering losses associated to inter-story drift-sensitive non structural components and losses to structural components). Based on the $\lambda_{a d m}$ and $L_{s c ~ a d m}$ values proposed for the limit states of DL and SD, the corresponding values of $E L_{a d m}$ set by Eq. (2) are then $10^{-4}$ and $5 \times 10^{-5}$, respectively.

\section{Results of the Probabilistic Performance Analysis}

\subsection{Initial Considerations}

Results of the seismic performance assessment carried out for the IRREG6 structure for the $\mathrm{DL}, \mathrm{SD}$, and NC limit states are presented in the following. To emphasize the influence of the modeling error of the selected capacity models, the performance results are presented for the case where the uncertainty of the component capacities is neglected and for the case where it is accounted for. For the DL limit state, the performance of the structure is analyzed considering $n_{L S}$ (the number of structural sections where the limit state mechanism occurs) values of one to six. For the SD limit state, the performance is analyzed considering $n_{L S}$ values of one to four instead. The mean and the standard deviation of the log of the data, which are required for Eqs. (5) and (6), were obtained using the logistic M-estimator proposed by Rousseeuw and Verboven [2002] and the $\tau$-scale truncated standard deviation proposed by Yohai and Zamar [1988], respectively. These estimators were selected based on the findings and recommendations presented by Romão et al. [2012a, b].

\subsection{Results for the DL Limit State}

The performance results of the structure are presented in Fig. 6. Parameters $\lambda_{U C}, L_{s c} U C$, and $E L_{U C}$ correspond to the values of $\lambda, L_{s c}$, and $E L$ obtained when the uncertainty of the component capacities is considered. The cumulative sum of $E L$ and $E L_{U C}$ up to each value of $n_{L S}$ along with the selected value for $E L_{a d m}$ are also represented. The results indicate that performance scenarios with $n_{L S}$ values higher than one can be seen to lead to acceptable values of $E L$. This simple comparison emphasizes the importance of using high-level parameters such as $\lambda, L_{s c}$ and $E L$ to analyze building performance. It is recalled that the

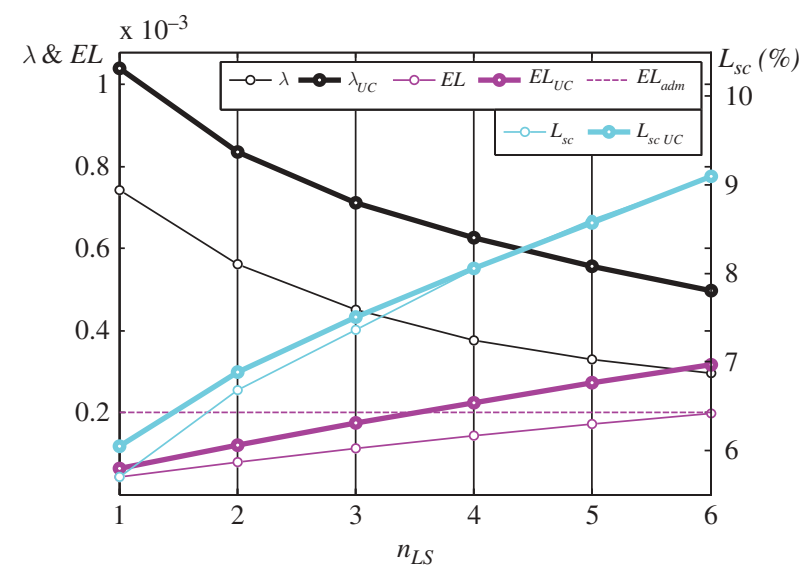

FIGURE 6 Results of the performance analysis of the IRREG6 structure. 
case where $n_{L S}=1$ corresponds to the scenario implicit in the code-based limit state definitions. Hence, by using $E L$ as a global performance measure (and accepting the values considered for $\lambda_{a d m}, L_{s c ~ a d m}$, and therefore $E L_{a d m}$ ), the proposed methodology is able to establish admissible performance scenarios that go beyond the limitations of the code definitions and allows for a rational decision-making process about the need to retrofit or strengthen a given structure.

According to the results, considering the uncertainty of the component capacities increases the risk considerably: $\lambda_{U C}$ is $40-70 \%$ larger than $\lambda$. On the other hand, the uncertainty effects on the expected costs are different. The uncertainty increases the expected costs for the $n_{L S}$ cases of one to three ( $L_{s c} U C$ is $6 \%$ larger than $L_{s c}$ when $n_{L S}=1$ ), while there is virtually no difference between $L_{s c} U C$ and $L_{s c}$ for the remaining $n_{L S}$ cases. In terms of the performance of the structure, the changes from $\lambda$ to $\lambda_{U C}$ and from $L_{s c}$ to $L_{s c} U C$ also modify the number of performance scenarios up to which the value of $E L$ is found to be admissible. As can be seen, the performance of the structure is only acceptable up to $n_{L S}=3$ when the uncertainty in the component capacities is considered. Therefore, this uncertainty component can be seen to have a significant influence on the acceptable performance of the structures, namely due to the significant increase of $\lambda$ to $\lambda_{U C}$.

The differences between $\lambda$ and $\lambda_{U C}$ can be explained by the analysis of the limit state fragility curves $p_{f, n_{L S}=i}$ presented in Fig. 7 a for the various $n_{L S}$ cases and for the cases where the uncertainty is and is not considered. When uncertainty is accounted for, the presented fragility curves indicate there is an increase in the variability of the data (i.e., there is a flattening of the curves) as well as a reduction in the median of the data. This reduction ranges from $1-5 \%$. Such shift in the median values of $S_{a, C}$ has also been reported by Liel et al. [2009], Vamvatsikos and Fragiadakis [2010], and Dolšek [2012], and can be seen to be the dominant factor leading to the higher values of $\lambda_{U C}$ since, according to Eq. (4), the higher values of $|d H(x) / d x|$ have now a larger contribution to $\lambda$.

Using a similar reasoning, the fragility curves $p_{\Delta_{C, 4}, n_{L S}=i}$ of the $\Delta_{C, 4}$ realizations of IRREG6 presented in Fig. 7b (which are similar to those obtained for the remaining stories) are also able to justify the differences between $L_{s c}$ and $L_{s c} U C$. When uncertainty is accounted for, these fragility curves show a small reduction in the median of the data (always lower than 5\%), but exhibit a significant increase in the variability of the data (between 60-270\%, depending on the story and $n_{L S}$ ). Since the considerable increase of
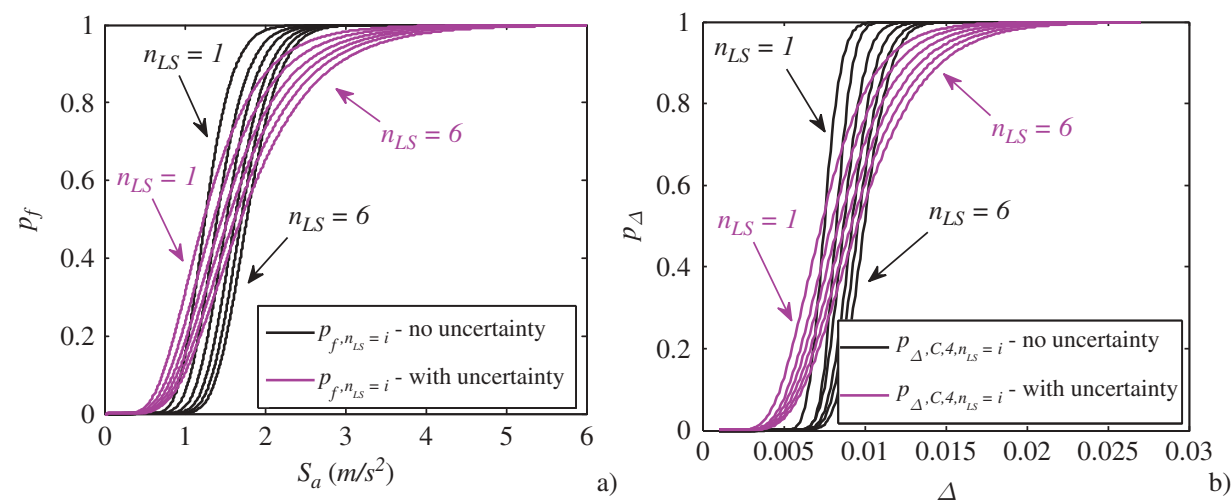

FIGURE 7 Fragility curves of the $S_{a, C}$ realizations (a) and fragility curves of the $\Delta_{C, 4}$ realizations (b), with and without the uncertainty of the component capacities and for $n_{L S}$ values of $1-6$. 
the dispersion of the data combined with the small changes in the median of the data only lead to a slight increase of $E L_{U C}$ for some $n_{L S}$ cases, this situation might not be statistically significant.

\subsection{Results for the SD Limit State}

The performance results are presented in Fig. 8 for the case where the uncertainty of the component capacities is neglected and also for the case where it is accounted for. In both cases, the performance is analyzed considering $n_{L S}$ values of one to four, and considering the chord rotation capacity defined by $\theta_{S D, 1}$.

As for the limit state of DL, the results show that including the uncertainty of the component capacities increases the risk considerably: $\lambda_{U C}$ is between $90-125 \%$ larger than $\lambda$. On the other hand, the influence of the uncertainty on the expected costs has an opposite effect: $L_{s c} U C$ is $11-12 \%$ lower than $L_{s c}$. The increase of $\lambda$ to $\lambda_{U C}$ and the reduction of $L_{s c}$ to $L_{s c} U C$ reflects the shift of the probabilistic distribution of the data due to the uncertainty, which has the direct effects of reducing the corresponding median values and increasing the variability of the data. The reduction of $L_{s c}$ to $L_{s c} U C$ can also be explained by analyzing the fragility curves $p_{\Delta_{C, j}, n_{L S}=i}$ of each story $j$. When uncertainty is accounted for, these fragility curves show a small reduction in the median of the data (always lower than 3\%) and exhibit an increase in the variability of the data (between 20-107\%, depending on the story and $\left.n_{L S}\right)$. Unlike for the DL limit state, $L_{s c} U C$ is governed by the reduction of the median since the increase in the variability is not large enough to counterbalance this effect.

As for the DL limit state, accounting for the uncertainty in the component capacities also modifies the number of performance scenarios up to which the value of $E L$ is found to be admissible. When the uncertainty in the component capacities is considered, the performance is acceptable up to $n_{L S}=3$. Although in this case the performance changes arise from the mixed effects of the increase in the risk and of the reduction of the expected costs, the significant increase of $\lambda$ to $\lambda_{U C}$ is still the governing factor.

As previously mentioned, the performance of the structure for this limit state was also carried out for the case where the chord rotation capacities are defined by $\theta_{S D, 2}$. This situation was considered in order to analyze the issue referred in Sec. 2.4.2 regarding the

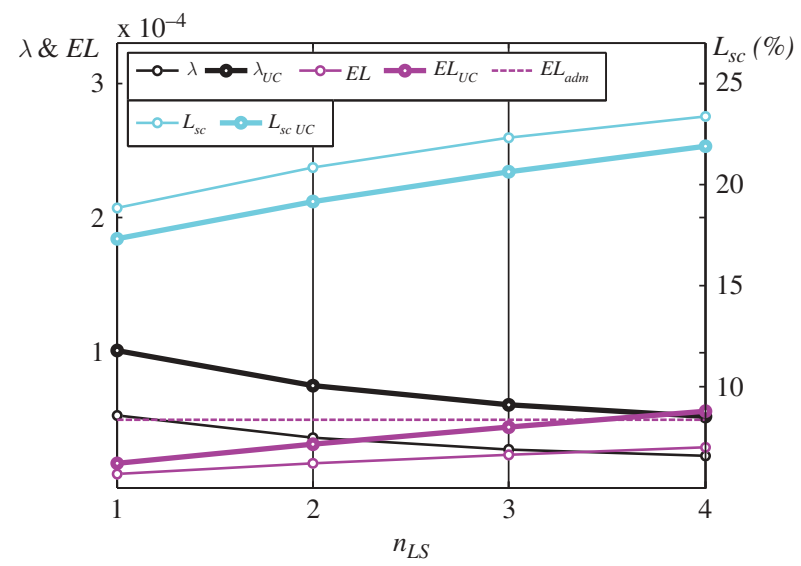

FIGURE 8 Results of the performance analysis of the IRREG6 structure when the chord rotation capacity is $\theta_{S D, 1}$. 
difference between the loss values of the case where a number of sections has $\rho$ values around $0.85-0.95$ and of the case where only one section has a $\rho$ value of 1.0. These two scenarios are considered to be comparable if similar $S_{a, C}$ realizations are required to activate them. Since it may be difficult to obtain a scenario with several sections having $\rho$ values around $0.85-0.95$ that matches the scenario where only one section has a $\rho$ value of 1.0, the scope of the analysis presented herein is extended. Therefore, scenarios where $n_{S D, 2}$ sections have $\rho$ values around $0.85-0.95$ are compared to scenarios where $n_{S D, 1}<n_{S D, 2}$ sections have a $\rho$ value of 1.0. In order to perform these comparisons, Fig. 9 presents the performance results of IRREG6 when the uncertainty of the component capacities is considered, and for the cases where the chord rotation capacity is $\theta_{S D, 1}$ and $\theta_{S D, 2}$. The results of $\lambda_{U C, 1}$ and $L_{s c} U C, 1$ refer to the cases where the chord rotation capacity is $\theta_{S D, 1}$, while the results of $\lambda_{U C, 2}$ and $L_{S c} U C, 2$ are those where the chord rotation capacity is $\theta_{S D, 2}$. As previously referred, the scenario comparison must be carried out for the $n_{L S}$ cases where $\lambda_{U C, 1} \approx \lambda_{U C, 2}$, i.e., performance scenarios for which the distribution of the $S_{a, C}$ realizations considering $\theta_{S D, 1}$ or $\theta_{S D, 2}$ is roughly the same. From the performance analysis of IRREG6, two sets of scenarios were seen to be similar. The first set of scenarios was found for $n_{L S}=1$, when $\theta_{S D, 1}$ is considered, and for $n_{L S}=2$, when $\theta_{S D, 2}$ is considered instead. To observe the similarity of the scenarios, Fig. 10 presents the fragility curves corresponding to their $S_{a, C}$ realizations. In this case, comparing the expected costs of the two scenarios shows that $L_{s c} U C, 2$ is only $2 \%$ larger than $L_{s c} U C, 1$. The second set of scenarios was found for $n_{L S}=2$, when $\theta_{S D, 1}$ is considered, and for $n_{L S}=4$, when $\theta_{S D, 2}$ is considered instead. In this case, comparing the expected costs of the two scenarios shows that $L_{s c} U C, 2$ is about $5 \%$ larger than $L_{s c} U C, 1$.

Based on these results, it is found that, for performance scenarios where the distribution of the $S_{a, C}$ realizations is similar, a lower limit state capacity occurring over a larger number of sections may lead to expected costs which are larger than those of the situation where a higher limit state capacity occurs over a smaller number of sections. Although these differences are not statistically significant, the observed trend should be further investigated given its potential influence in one of the performance metrics considered in the proposed methodology.

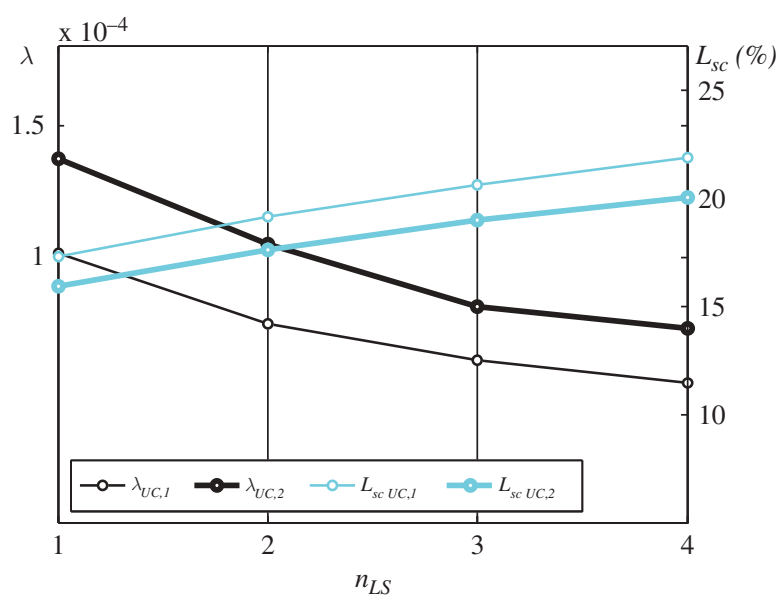

FIGURE 9 Results of the performance analysis of the IRREG6 structure when the uncertainty of the component capacities is considered, and the chord rotation capacity is $\theta_{S D, 1}$ and $\theta_{S D, 2}$. 


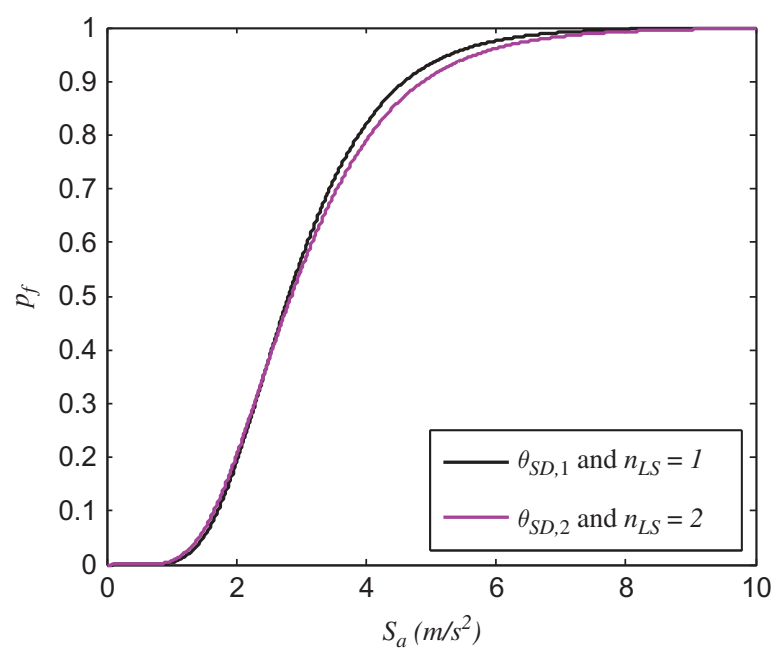

FIGURE 10 Similarity of the fragility curves of the $S_{a, C}$ realizations obtained for different $n_{L S}$ values when the chord rotation capacity is $\theta_{S D, 1}$ and $\theta_{S D, 2}$.

\subsection{Results for the NC Limit State}

Given the assumptions established in Sec. 2.4.3, only $\lambda$ values are presented to analyze the performance of the IRREG6 structure for the limit state of NC. With respect to the limit state conditions also defined in Sec. 2.4.3, the occurrence of a NC limit state nonconforming condition in all the beam sections of a given story was not a governing scenario in any of the cases analyzed, both in terms of the rotation capacity $\theta_{N C}$ and of the shear force capacity $V_{N C}$. Furthermore, the occurrence of the shear force capacity $V_{N C}$ in columns was not a governing scenario also. Hence, the NC limit state performance of the structures was governed by the occurrence of the NC rotation capacity in columns and by the development of global yield mechanisms. In order to observe the importance of each of these nonconforming conditions, the following five scenarios were analyzed for this limit state.

Scenario 1. Only the column rotation demand is controlled and the uncertainty of the rotation capacities is not considered $\left(S_{\theta_{N C}}\right)$.

Scenario 2. Only the column rotation demand is controlled and the uncertainty of the rotation capacities is accounted for $\left(S_{\theta_{N C, U}}\right)$.

Scenario 3. Only the development of global yield mechanisms is controlled $\left(S_{G Y M}\right)$.

Scenario 4. Both the column rotation demand and the development of global yield mechanisms are controlled and the uncertainty of the rotation capacities is not considered $\left(S_{\theta_{N C}}+S_{G Y M}\right)$.

Scenario 5. Both the column rotation demand and the development of global yield mechanisms are controlled and the uncertainty of the rotation capacities is accounted for $\left(S_{\theta_{N C, U}}+S_{G Y M}\right)$.

Based on these scenarios, it is reminded that the uncertainty in the development of the global yield mechanisms due to the uncertainty in the value of the yield curvature of the components has not been considered. The $\lambda$ values which correspond to the performance results of IRREG6 for the five scenarios are presented in Table 2. The presented results 
TABLE 2 Performance results of IRREG6 for the NC limit state considered scenarios

\begin{tabular}{lc}
\hline Scenario & $\lambda$ - IRREG6 \\
\hline$S_{\theta_{N C}}$ & $4.95 \mathrm{E}-5$ \\
$S_{\theta_{N C, U}}$ & $7.02 \mathrm{E}-5$ \\
$S_{G Y M}$ & $1.91 \mathrm{E}-4$ \\
$S_{\theta_{N C}}+S_{G Y M}$ & $1.90 \mathrm{E}-4$ \\
$S_{\theta_{N C, U}}+S_{G Y M}$ & $2.00 \mathrm{E}-4$ \\
\hline
\end{tabular}

indicate that only scenarios that do not involve the development of global yield mechanisms are able to conform to the condition $\lambda \leq \lambda_{\text {adm }}=0.0001$. As can be observed, when the development of global yield mechanisms is considered, the $\lambda$ values almost duplicate. This fact clearly emphasizes the importance of considering this type of condition when analyzing structural safety and performance under earthquake loading.

When considering the scenario $S_{\theta_{N C}}+S_{G Y M}$, the analysis of the results indicates that the limit state capacity was governed by the rotation demand in a column for only one ground motion. This situation implies that the median of the $S_{a, C}$ realizations has a $0.2 \%$ reduction from the scenario $S_{G Y M}$ to the scenario $S_{\theta_{N C}}+S_{G Y M}$ and that the standard deviations of the $\log$ of the $S_{a, C}$ realizations has a reduction of about $2.7 \%$. The latter reduction is the governing factor and leads to the slight decrease of the $\lambda$ value from $S_{G Y M}$ to $S_{\theta_{N C}}+S_{G Y M}$. When comparing the scenarios $S_{G Y M}$ and $S_{\theta_{N C, U}}+S_{G Y M}$, the uncertainty in the rotation capacities plays a larger role and reduces the median of the $S_{a, C}$ realizations by $1.9 \%$. Although there is also a $1.9 \%$ reduction of the standard deviations of the log of the $S_{a, C}$ realizations from $S_{G Y M}$ to $S_{\theta_{N C, U}}+S_{G Y M}$, the shift of the median is now the governing factor leading to the increase of the $\lambda$ value from $S_{G Y M}$ to $S_{\theta_{N C, U}}+S_{G Y M}$. Although the mentioned reductions explain the variability of $\lambda$ between scenarios $S_{G Y M}, S_{\theta_{N C}}+S_{G Y M}$ and $S_{\theta_{N C, U}}+S_{G Y M}$, they are not seen to be statistically significant since the overall changes in $\lambda$ are below $5 \%$.

With respect to the global yield mechanisms that were found when analyzing this limit state, the unpredictability of their configurations and the importance of using a technique such as the one presented in Sec. 2.4.3 should be emphasized. In order to illustrate some of the global yield mechanisms that were found, Fig. 11 presents two examples. Although
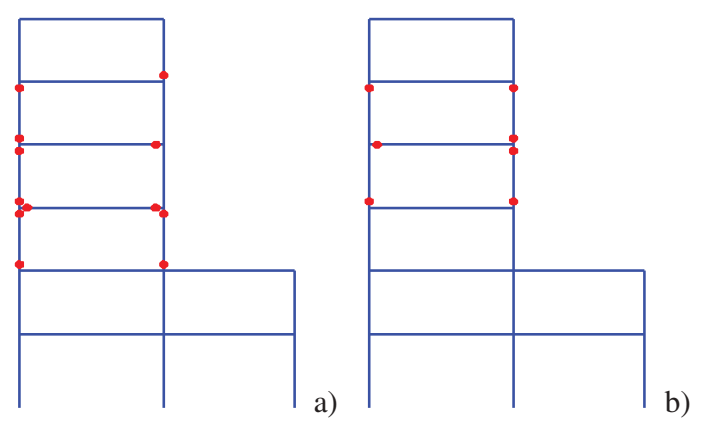

FIGURE 11 Examples of global yield mechanism configurations that were found when analyzing the NC limit state. (C) Nova Science Publishers, Inc. Reproduced by permission of Nova Science Publishers, Inc. Permission to reuse must be obtained from the rightsholder. 
the case presented in Fig. 11a ended up being controlled by a familiar mechanism (a soft-story mechanism), the case of Fig. $11 \mathrm{~b}$ is less common. These results indicate that approaches such as the one referred by Jalayer et al. [2007] that require the identification of the global yield mechanism configurations may not be practical to use due to the multitude of possibilities.

\section{Conclusion}

A probabilistic methodology was proposed to analyze the seismic performance of existing buildings using global metrics to determine if the behavior conforms to a given limit state. The considered performance metrics are the mean annual frequency $\lambda$ of the limit state, the corresponding loss $L_{s c}$ associated to the repair of the building, and the corresponding number $n_{L S}$ and type of mechanisms that occur. Each case of $n_{L S}$ establishes a scenario corresponding to the occurrence of the limit state. The $\lambda$ and $L_{s c}$ values of each scenario are then combined to define a global performance parameter representing the expected loss $E L$ associated to that limit state. To be able to consider these performance metrics, an update of existing limit state descriptions was performed. Alternative proposals were discussed to establish risk- and cost-related limit state definitions based on those proposed by EC8-3.

An application of the methodology was presented which involved the seismic performance analysis of a RC structure for several limit states and included the analysis of the effects of the modelling error of the structural component capacities. The performance results indicate the uncertainty of the component capacities increases the risk considerably while leading to small reductions of the expected losses. Accounting for the uncertainty of the component capacities was found to produce a shift of the probabilistic distribution of the data. As a direct effect of such shift, there is a reduction in the median of the data, which was found to be the governing factor for the increase and the reduction in the risk and expected loss values, respectively. Moreover, such results are seen to disagree with the conventional assumption that accounting for modeling uncertainties has the unique effect of increasing the dispersion of the fragility curves without influencing the median of the data (a fact also disputed by the findings reported by Liel et al. [2009], Vamvatsikos and Fragiadakis [2010], and Dolšek [2012]).

Overall, the proposed methodology was able to determine admissible performance scenarios that go beyond the limitations of the code definitions and allow for a rational decision-making process about the need to retrofit or strengthen a given structure. In particular, the performance analysis carried out for the NC limit state emphasized the importance of considering the potential occurrence of global yield mechanisms, as well as that of having a process able to account for the unpredictability of their configurations.

\section{Acknowledgments}

Figures 1-5 and 11 are reprinted with permission from Nova Science Publishers, Inc. from "A risk- and cost-based methodology for the seismic safety assessment of existing buildings" in Earthquakes: Risk Factors, Seismic Effects and Economic Consequences, by Romão, X., Delgado, R. and Costa, A., copyright (2014).

\section{Funding}

Financial support of the Portuguese Foundation for Science and Technology, through the research grant PTDC/ECM/108098/2008 (Development and calibration of seismic safety assessment methodologies for existing buildings according to the Eurocode 8 - Part 3), is gratefully acknowledged. 


\section{References}

Ambraseys, N., Simpson, K., and Bommer, J. [1996] "Prediction of horizontal response spectra in Europe," Earthquake Engineering Structural Dynamics 25(4), 371-400.

ASCE [2003] Seismic Evaluation of Existing Buildings (ASCE/SEI 31-03). American Society of Civil Engineers, Reston, Virginia.

ASCE [2007] Seismic Rehabilitation of Existing Buildings (ASCE/SEI 41-06). American Society of Civil Engineers, Reston, Virginia.

ATC [1996] ATC 40 Seismic Evaluation and Retrofit of Concrete Buildings. Applied Technology Council, Redwood City, California.

ATC [2009] ATC-63 Quantification of Building System Performance and Response Parameters. Applied Technology Council, Redwood City, California.

Bradley, B. and Dhakal, R. [2008] "Error estimation of closed-form solution for annual rate of structural collapse," Earthquake Engineering and Structural Dynamics 37(15), 1721-1737.

Campos Costa, A., Sousa, M., and Carvalho, A. [2008] "Seismic zonation for Portuguese National Annex of Eurocode 8." Proc. of the 14th World Conference on Earthquake Engineering. Beijing, China.

CEN [2005] EN 1998-3 Eurocode 8: Design of Structures for Earthquake Resistance, Part 3: Assessment and Retrofitting of Buildings. European Committee for Standardization.

Deierlein, G. and Haselton, C. [2005] "Benchmarking the collapse safety of code compliant reinforced concrete moment frame building systems." ATC/JSCA US-Japan Workshop on Improvement of Structural Design and Construction Practices, Proc. of an International Workshop, Kobe, Japan.

Deierlein, G., Liel, A., Haselton, C., and Kircher, C. [2008] "ATC-63 methodology for evaluating seismic collapse safety of archetype buildings." Proc. of the ASCE-SEI Structures Congress, Vancouver, Canada.

Diamantidis, D. and Bazzurro, P. [2007] "Safety acceptance criteria for existing structures," Workshop on Risk Acceptance and Risk Communication, Stanford University, Stanford, California.

DOE [2002] Natural Phenomena Hazards Design and Evaluation Criteria for Department of Energy Facilities. DOE-STD-1020-2002. U.S. Department of Energy, Washington D.C.

Dolšek, M. [2012] "Simplified method for seismic risk assessment of buildings with consideration of aleatory and epistemic uncertainty," Structure and Infrastructure Engineering 8(10), 939-953.

EC8-1 [2009] NP ENV 1998-1. Portuguese National Annex to Eurocode 8: Design of Structures for Earthquake Resistance - Part 1: General Rules, Seismic Actions and Rules for Buildings. Instituto Português da Qualidade, Caparica, Portugal.

Fajfar, P. and Dolšek, M. [2012] "A practice-oriented estimation of the failure probability of building structures," Earthquake Engineering and Structural Dynamics 41(3), 531-547.

Ferracuti, B., Pinho, R., Savoia, M., and Francia, R. [2009] "Verification of displacement-based adaptive pushover through multi-ground motion incremental dynamic analyses," Engineering Structures 31(8), 1789-1799.

fib [2003a] "Seismic assessment and retrofit of reinforced concrete buildings," Fédération Internationale du Béton, Bulletin $\mathrm{n}^{\circ} 24$.

fib [2003b] "Displacement-based seismic design of reinforced concrete buildings," Fédération Internationale du Béton, Bulletin $\mathrm{n}^{\circ} 25$.

Foltz, R. [2004] "Estimating seismic damage and repair costs," Proc. of the 2004 Earthquake Engineering Symposium for Young Researchers. Charleston, South Carolina.

Haselton, C. [2006] "Assessing seismic collapse safety of modern reinforced concrete moment frame buildings," Ph.D. Dissertation, Dept. of Civil and Environmental Engineering, Stanford University. Stanford, California.

Haselton, C., Liel, A., Deierlein, G., Dean, B., and Chou, J. [2011] "Seismic collapse safety of reinforced concrete buildings. I: Assessment of ductile moment frames," Journal of Structural Engineering 137(4), 481-491. 
Ibarra, L. and Krawinkler, H. [2005] "Global collapse of frame structures under seismic excitations," Report No. PEER 2005/06, Pacific Earthquake Engineering Research Center, University of California at Berkeley, California.

Ibarra, L., Medina, R., and Krawinkler, H. [2002] "Collapse assessment of deteriorating SDOF systems," Proc. of the 12th European Conference on Earthquake Engineering, London.

ISO 13822 [2010] Bases for Design of Structures — Assessment of Existing Structures, International Organization for Standardization, Geneva, Switzerland.

ISO 2394 [1998] General Principles on Reliability for Structures. International Organization for Standardization, Geneva, Switzerland.

Jalayer, F., Franchin, P., and Pinto, P. E. [2007] "A scalar damage measure for seismic reliability analysis of RC frames," Earthquake Engineering and Structural Dynamics 36(13), 2059-2079.

JCSS [2001] Probabilistic Assessment of Existing Structures. Joint Committee on Structural Safety. RILEM Publications S.A.R.L.

Kircher, C. and Heintz, J. [2008] "Overview and key concepts of the ATC-63 methodology," Proc. of the ASCE-SEI Structures Congress, Vancouver, Canada.

Krawinkler, H. and Lignos, D. [2009] "How to predict the probability of collapse of non-ductile building structures," in Seismic Risk Assessment and Retrofitting, Geotechnical, Geological, and Earthquake Engineering, (Springer: New York), Ch. 17, 10, 343-365.

Liel, A., Haselton, C., Deierlein, G., and Baker, J. [2009] "Incorporating modeling uncertainties in the assessment of seismic collapse risk of buildings," Structural Safety 31(2), 197-211.

Liel, A., Haselton, C., and Deierlein, G. [2011] "Seismic collapse safety of reinforced concrete buildings. II: Comparative assessment of nonductile and ductile moment frames," Journal of Structural Engineering 137(4), 492-502

Nafday, A. [2008] "System safety metrics for skeletal structures," Journal of Structural Engineering 134(3), 499-504.

NTC [2008] Norme Tecniche per le Costruzioni. Decreto del Ministero delle infrastrutture, Supplemento Ordinario n.30 alla Gazzetta Ufficiale della Repubblica italiana n.29 del 4/02/2008, Italy (in Italian).

NZSEE [2006] "Assessment and Improvement of the Structural Performance of Buildings in Earthquake," Recommendations of a NZSEE Study Group on Earthquake Risk Buildings. New Zealand Society for Earthquake Engineering, New Zealand.

PEER-NGA [2009] NGA Database, Pacific Earthquake Engineering Research Center; http://peer. berkeley.edu/nga/. Accessed April 2010.

Pinto, P. E., Giannini, R., and Franchin, P. [2004] Seismic reliability analysis of structures. IUSS Press, Istituto Universitario di Studi Superiori di Pavia: Pavia, Italy.

Ramirez, C. and Miranda, E. [2009] "Building-specific loss estimation methods \& tools for simplified performance-based earthquake engineering.” Report No. 171. John A. Blume Earthquake Engineering Research Center. Stanford University. Stanford, California.

Romão, X., Costa, A., and Delgado, R. [2011] "Assessment of the statistical distributions of structural demand under earthquake loading," Journal of Earthquake Engineering 15(5), 724-753.

Romão, X., Costa, A., and Delgado, R. [2012a] "Statistical characterization of structural demand under earthquake loading. Part 1: Robust estimation of the central value of the data," Journal of Earthquake Engineering 16(5), 686-718.

Romão, X., Costa, A., and Delgado, R. [2012b] "Statistical characterization of structural demand under earthquake loading. Part 2: Robust estimation of the dispersion of the data," Journal of Earthquake Engineering 16(6), 864-896.

Rousseeuw, P. and Verboven, S. [2002] "Robust estimation in very small samples," Computational Statistics and Data Analysis 40(4), 741-758.

Sousa, M. and Oliveira, C. [1996] "Hazard mapping based on macroseismic data considering the influence of geological conditions," Natural Hazards 14(2), 207-225.

Veneziano, D., Casciati, F., and Faravelli, L. [1983] "Method of seismic fragility for complicated systems," Proc. of the 2nd Specialist Meeting on Probabilistic Methods in Seismic Risk Assessment for NPP, Committee on Safety of Nuclear Installations (CSNI), Livermore, California. 
Vamvatsikos, D. and Cornell, C. A. [2002] "Incremental dynamic analysis," Earthquake Engineering and Structural Dynamics 31(3), 491-514.

Vamvatsikos, D. and Fragiadakis, M. [2010] "Incremental dynamic analysis for seismic performance uncertainty estimation," Earthquake Engineering and Structural Dynamics 39(2), 141-163.

Vrouwenvelder, T. and Scholten, N. [2010] "Assessment criteria for existing structures," Structural Engineering International 20(1), 62-65.

Welch, D., Sullivan, T., and Calvi, G. [2014] "Developing direct displacement-based procedures for simplified loss assessment in performance-based earthquake engineering," Journal of Earthquake Engineering 18(2), 290-322.

Wen, Y. and Ellingwood, B. [2005] "The role of fragility assessment in consequence-based engineering," Earthquake Spectra 21(3), 861-877.

Yohai, V. and Zamar, R. [1988] "High breakdown-point estimates of regression by means of the minimization of an efficient scale," Journal of the American Statistical Association 83(402), 406-413.

Zareian, F. and Krawinkler, H. [2007] "Assessment of probability of collapse and design for collapse safety,” Earthquake Engineering and Structural Dynamics 36(13), 1901-1914. 